\title{
Making classical and quantum canonical general relativity computable through a power series expansion in the inverse cosmological constant
}

\author{
Rodolfo Gambini ${ }^{1}$, Jorge Pullin ${ }^{2}$ \\ 1. Instituto de Fúsica, Facultad de Ciencias, Iguá 4225, esq. Mataojo, Montevideo, Uruguay. \\ 2. Center for Gravitational Physics and Geometry, Department of Physics, \\ The Pennsylvania State University, 104 Davey Lab, University Park, PA 16802.
}

(August 14th 2000)

\begin{abstract}
We consider general relativity with a cosmological constant as a perturbative expansion around a completely solvable diffeomorphism invariant field theory. This theory is the $\Lambda \rightarrow \infty$ limit of general relativity. This allows an explicit perturbative computational setup in which the quantum states of the theory and the classical observables can be explicitly computed. The zeroth order corresponds to highly degenerate space-times with vanishing volume. Perturbations give rise to space-times with non-vanishing volumes in a natural way. The spectrum of area- and volume-related observables constructed by coupling the theory to matter can be directly assessed. An unexpected relationship arises at a quantum level between the discrete spectrum of the volume operator and the allowed values of the cosmological constant.
\end{abstract}

The recent years have been very fertile for the development of canonical quantizations of general relativity. There are now available more than one [1,2] highly non-trivial mathematically consistent, finite, anomalyfree theories of canonical quantum gravity. Instrumental in these developments have been the underlying advances in mathematical techniques for dealing with infinite dimensional nonlinear spaces, like the theory of cylindrical functions and associated measures [3], and the introduction of spin networks to eliminate the over-completeness of the Wilson loop basis 价 (for a recent summary, see the review article by Rovelli [5]). In spite of the advances, most of the results obtained from these theories up to now concern statements made at a kinematical level (without imposing the Hamiltonian constraint). One of the remaining main challenges is to obtain results that hold at a dynamical level, imposing the full set of constraints of the theory. This requires making the theories computable, in the sense of obtaining classical observables to promote to quantum observables and solving for the physical set of states that are annihilated by all the constraints. Only in this setting will one be able to make contact with trustworthy physical predictions.

The fact that implementing the full dynamics of canonical quantum gravity is hard to do has plagued the subject since its inception in the 60's by DeWitt [6]. For instance, one does not know even at a classical level how to compute observable quantities for canonical general relativity (quantities that are invariants under the symmetries of the theory or - in the canonical language - that commute with the constraints), and it is known that such quantities are potentially quite involved $[7]$. As a consequence, it is very hard to make contact with the semiclassical limit, since one does not have at hand observable quantities to compute. Moreover, the space of quantum states that are annihilated by the quantum constraints cannot be straightforwardly computed [8]. None of these issues was directly tackled by the significant amount of progress that was made in terms of the introduction of connection type variables and loop representations for quantum gravity that we mentioned at the beginning. There is general consensus that an approximation scheme is desirable in such a way as to make the above problems solvable. The challenge consists in finding an approximation scheme that will not conflict with non-perturbative nature of the canonical quantization, in particular the diffeomorphism invariance of the theory.

In this paper we would like to propose a computational scheme that allows to explicitly address the issue of finding observables for the theory (at a classical level) and to find quantum states annihilated by all the constraints. The central idea will be to consider general relativity as a power series expansion in terms of the inverse cosmological constant, and to view the full theory as a "perturbation" of a zeroth order solvable theory. The zeroth order theory corresponds to general relativity in the limit in which the cosmological constant $\Lambda \rightarrow \infty$. The use of an approximation based on a large cosmological constant has been pioneered in a different context (that of studying the spectrum of the physical Hamiltonian obtained by "de-parameterizing" the theory) by Smolin [9] and also by Rovelli [10], with some level of success. We will however be able to make further progress, in significant part due to the new maturity of the field we mentioned above but also because we push the perturbative approach at all levels: constraints, observables and equations of motion, both classically and quantum mechanically. It might be argued that a scheme where the cosmological constant is large (in Planck units) lacks physical relevance, given is small present value. However, having a regime of the theory in which it is completely solvable can be a valuable tool, even if such regime is unphysical. Moreover, as we will discuss below, there is some evidence that our approach might be useful beyond its apparent domain of validity. It might be the case that the situation is analogous to the large $N$ approximation in QCD or the strong 
coupling expansions of lattice field theory, that a priori look less promising that what they end up being.

The starting point is to consider canonical quantum gravity with a cosmological constant. The Hamiltonian constraint of the theory is,

$$
H(N)=H_{0}(N)+\Lambda \int d^{3} x N(x) \sqrt{\operatorname{det} q(x)}
$$

where $N(x)$ is a smearing function, $H_{0}(N)$ is the Hamiltonian constraint without a cosmological constant and $\operatorname{det} q$ is the determinant of the spatial metric. If one now considers the limit $\Lambda \rightarrow \infty$, and re-scales the constraint by $1 / \Lambda$ [1] one is left with a theory, which we will call "zeroth order" theory for which the Hamiltonian constraint is, $H^{(0)}(N)=\int d^{3} x N(x) \sqrt{\operatorname{det} q(x)}$, that is, the Hamiltonian constraint is just the square root of the determinant of the spatial metric. In addition to this the theory has the ordinary diffeomorphism constraint and if one uses Ashtekar variables there will also be a Gauss law constraint (both are independent of $\Lambda$ ). Imposing classically the Hamiltonian constraint of the zeroth order theory, one immediately sees that it corresponds to metrics of identically vanishing determinant, that is, degenerate metrics.

The dynamics of the zeroth order theory is easily solved. One can readily construct quantities that have vanishing Poisson bracket with the Hamiltonian constraint of the zeroth order theory. For instance, one can consider any function depending only on the three metric (or if one is using Ashtekar variables $\left(\tilde{E}_{i}^{a}, A_{b}^{j}\right)$, the densitized triads $\tilde{E}_{i}^{a}$ ) and not on its canonically conjugate momenta. To be a genuine observable the quantity should also have vanishing Poisson bracket with the diffeomorphism constraint. A way of constructing quantities with this property is to couple the theory with matter fields and physically define geometric objects like surfaces or volumetric regions through properties of the matter fields 12,13 and then compute the areas or volumes of these regions. The usual problem with this construction is that the addition of matter adds terms to the Hamiltonian constraint as well, and the areas and volumes computed as we just described do not commute with the Hamiltonian constraint. However, in the $\Lambda \rightarrow \infty$ limit the matter terms drop out of the Hamiltonian and one ends up (in the zeroth order theory) with genuine observables (this is similar to what happens in models without a Hamiltonian constraint as was discussed by Rovelli in the HusainKuchař [11] model 10]). A concrete example of an observable for the zeroth order theory is obtained by coupling the theory to an antisymmetric tensor field with the standard Kalb-Ramond free Hamiltonian as proposed by Smolin 13]. The antisymmetric tensor field is naturally associated with surfaces of which one can compute the area. The resulting quantity, which essentially is a contraction of the triads with the canonically conjugate momenta of of the Kalb-Ramond field, is naturally diffeo- morphism invariant. One can build a "loop-surface" representation (see 13,14 for details) for the coupled theory by expanding the gauge invariant wavefunctions in a basis of states $\mid \Sigma, s>$ labeled by equivalence classes under diffeomorphisms of spin networks $s$ and three-surfaces $\Sigma$. A natural generalization of the Ashtekar-Lewandowski [3] measure allows to introduce an inner product. On this basis the area defined by the fields has a discrete spectrum.

The Hamiltonian constraint of the zeroth order theory can be readily realized in the kinematical space [4, 15] of non-diffeomorphism invariant wavefunctions as,

$$
H^{(0)}(N)\left|\Sigma, s>=\lim _{\epsilon \rightarrow 0} \sum_{v \in s} N(v) V(v, \epsilon)\right| \Sigma, s>
$$

where we have represented, following Thiemann [1], the determinant of the metric as the volume operator of an infinitesimal region of size $\epsilon$ centered on the vertices $v$ of the spin network. The operator is independent on $\epsilon$ on this space of states and therefore the above limit is well defined. To consider the action on diffeomorphism invariant states one evaluates the inner product $<\left\{\Sigma^{\prime}\right\},\{s\}\left|H^{(0)}(N)\right| \Sigma, s>$ which is well defined. We can readily find the space of states that are annihilated by the Hamiltonian constraint of the zeroth order theory: they correspond to states based on spin networks such that the localized volume operator vanishes on each of their vertices. For trivalent vertices this is the case for all states. For four and higher valent vertices the operator vanishes automatically if the vertices are planar (this is not true for the volume operator of [4], but it holds for the definition of [15). If the vertices are not planar it is also possible to find values of the valences of the incoming edges such that the operator vanishes. We collectively denote all these states as $\left|\Sigma, s^{0}\right\rangle$. Since the space of solutions of the Hamiltonian constraint is a subspace of the states we were considering, it can be readily endowed with an inner product which is just the restriction to this space of the inner product derived from the Ashtekar-Lewandowski measure. Notice that the area observables we introduced are well defined on this space.

We have therefore seen that the zeroth order theory is well defined. It is non-perturbatively quantized in a basis of spin networks and surfaces, one can solve for the physical states, construct observables, and introduce a physical inner product. This is therefore a sound starting point for a perturbative expansion. The resulting perturbative theory is remarkably similar to bound state perturbation theory in quantum mechanics in the degenerate case. The operator $H^{(0)}$ (which can be readily diagonalized in the basis of states considered) has a degenerate spectrum.

To consider the higher order corrections to the zeroth order theory, one is interested in solving the eigenvalue problem

$$
<\phi\left(\Lambda^{-1}\right) \mid H^{(0)}+\Lambda^{-1} H^{(1)}=\epsilon(\Lambda)<\phi\left(\Lambda^{-1} \mid\right.
$$


with eigenvalue $\epsilon(\Lambda)$ equal to zero up to the desired order in perturbation theory. We will assume that the states and the eigenvalue can be expanded in power series in the inverse cosmological constant, and address the resulting equations order by order. Since the spectrum of the zeroth order Hamiltonian is degenerate, we apply degenerate perturbation theory to solve this problem. One starts by considering a state with eigenvalue $\epsilon^{(0)}$ for the zeroth order Hamiltonian constraint, that is, a spin network state with a definite volume. Notice that this is not a state of the zeroth order theory. Considering first order corrections and solving the equations resulting from (3) one determines the first order corrections and a polynomial $\epsilon\left(\Lambda^{-1}\right)$ which, set to zero, determines the value of $\Lambda$ for which the perturbative treatment is valid. That is, given an eigenvalue of the zeroth order Hamiltonian, one constructs a solution of the first order theory that has vanishing Hamiltonian for a particular value of lambda. That is, as a consequence of the discrete spectrum of the zeroth order Hamiltonian inherited from the discreteness of the volume operator, the cosmological constant is, in this approach, quantized.

This might sound unusual, but imposing the constraint perturbatively is analogous to considering an atom in a magnetic field and trying to find the states with a given energy level. Such energy level can be reached by choosing a nearby energy state of an unperturbed atom and then tuning the magnetic field to a given value. Various states could correspond to the same energy, depending on the value of the magnetic field. The approximate value of the magnetic field required will also depend on how high one goes in perturbation theory. A detailed discussion of how this is implemented in several systems can be seen in 16 .

A remarkable aspect is that all the calculations involved in the perturbative approach are completely explicit when one works in the spin network basis. One starts by considering eigenvalues and eigenvectors of the zeroth order Hamiltonian. Since this Hamiltonian is the volume operator, which is well understood, the space of states is under control (even when it is non-vanishing). The equation that determines the first order correction involves evaluating the Hamiltonian constraint on the states with a given volume. Such calculations are completely explicit given, for instance, the Hamiltonian introduced by Thiemann [1], or the Hamiltonian in the Vassiliev arena [2]. The only subtle element in the calculation is that given the infinite degeneracy of the volume operator, the first order correction might involve infinite superpositions and therefore may lead us to corrections to the kinematical inner product. It is worthwhile noticing that in general the first order corrections have non-zero volume, even if one started from the zeroth order state with vanishing volume, as long as one did not restrict to either planar or trivalent spin networks. So the resulting theory is a theory of non-vanishing volume space-times.
The above construction has a classical counterpart when one tries to find observables for the theory. An observable for the first order theory can be written as a perturbation of the observables of the zeroth order level theory we discussed above,

$$
O_{\Lambda}(\pi, \tilde{E})=O^{(0)}(\pi, \tilde{E})+\Lambda^{-1} O^{(1)}(\pi, \tilde{E}) .
$$

One would like to request that these observables have vanishing Poisson brackets with the Hamiltonian of the theory (more precisely, weakly vanishing, but the extension to such case is immediate). Expanding such requirement in powers of $\Lambda^{-1}$ one gets to zeroth and first order in $\Lambda^{-1}$,

$$
\begin{array}{r}
\left\{O^{(0)}, H^{(0)}\right\}=0 \\
\left\{O^{(1)}, H^{(0)}\right\}+\left\{O^{(0)}, H^{(1)}\right\}=0
\end{array}
$$

The first equation determines $O^{(0)}$ and the second one leads to a (functional) linear partial differential equation for $O^{(1)}$. The construction can be readily continued to higher orders. In all cases one obtains a linear partial differential equation, albeit with a more and more complex inhomogeneous term. It should be noted that one can obtain many observables starting with different solutions to the first equation. The linear partial differential equations are not hard to solve, given that the coefficients of the derivatives are functions of the triads, whereas the derivatives are with respect to the connections. Given its simplicity, the system is always integrable and therefore yields all the observables of the theory. The solutions to these equations will generically be ill defined or non-convergent in certain regions of phase space. This is expected in theories with complicated dynamics and is not a pathology of the method per se but rather a feature of the observable quantities. In particular, one might be concerned that the current approach produces too many observables. For instance, there exist pathological systems that have less observables than degrees of freedom (e.g. the heavy asymmetric top). A priori our method seems to yield in these cases extra observables, but on closer examination the quantities constructed are not acceptable (in the case of the top they take values on a restricted portion of phase space not preserved by evolution). We discuss several pathological examples and how the method correctly handles them in [16].

Let us consider, as an simple example of interest in gravity, the computation of an observable for Bianchi cosmologies. Observables for these models (with cosmological constant) had never been studied before. We will write the Bianchi models in terms of Ashtekar's variables following the notation of 17], in which one codes all the information in three variables associated with the time dependent portion of the triads $E^{1}, E^{2}, E^{3}$ and their conjugate momenta $A_{1}, A_{2}, A_{3}$. The only constraint of the 
theory given the homogeneity and diagonality of the metric is the Hamiltonian, which rescaled by the cosmological constant reads,

$$
H=E^{1} E^{2} E^{3}+\Lambda^{-1}\left(E^{1} E^{2}\left(A_{1} A_{2}-\epsilon A_{3}\right)+\text { cyclic }\right),
$$

where $\epsilon=1$ corresponds to the Bianchi IX model and $\epsilon=0$ to the Bianchi I model and the extra terms are obtained by cyclically permuting the indices $1,2,3$. It is straightforward to repeat the construction for the other class-A Bianchi models.

The observables of the zeroth order theory are given by functions $O^{0}=F\left(E^{1}, E^{2}, E^{3}, E^{1} A_{1}-E^{2} A_{2}, E^{1} A_{1}-\right.$ $E^{3} A_{3}$ ) (if f one considers $F\left(E^{1} A_{1}-E^{2} A_{2}, E^{1} A_{1}-E^{3} A_{3}\right.$ ) these objects are exact observables to all orders for the Bianchi I case).

As an example, we can start with an observable of the zeroth order theory $O^{(0)}=E^{1}$. The condition (6) translates into the following partial differential equation

$$
\frac{\partial O^{(1)}}{\partial A_{1}} E^{2} E^{3}-E^{1} E^{2} A_{2}-E^{1} E^{3} A_{3}+\epsilon E^{2} E^{3}+\text { cyclic }=0 .
$$

This yields a first order correction, which modulo the Hamiltonian constraint can be written as,

$$
O_{1}^{(1)}=-\frac{A_{1}}{E^{2} E^{3}}\left(-E^{1} E^{2} A_{2}+\left(E^{1}\right)^{2} A_{1}-E^{1} E^{3} A_{3}\right),
$$

up to an arbitrary additive function $F\left(E^{1}, E^{2}, E^{3}, E^{1} A_{1}\right.$ $\left.E^{2} A_{2}, E^{1} A_{1}-E^{3} A_{3}\right)$ that solves the homogeneous part of the equation. Expressions for higher orders can easily be generated using computer algebra, we do not list them here for reasons of space. The behavior of the approximate observables can be studied explicitly using the exact solution [18] of the equations of motion (in the Bianchi I case). One observes that, evaluated on the trajectories of the solution, the coefficients in the power series expansion lose their dependence on the cosmological constant as an expansion parameter. Carefully choosing the arbitrary functions $F$ one can define observables that are finite at the Big Bang and remain approximately constant for a significant portion of the lifetime of the (recollapsing) universe. The rate of change decreases if one adds further terms in the expansion, exhibiting convergence, although all information about $\Lambda$ has disappeared. Therefore quite unexpectedly, the method produces solutions that are accurate irrespective of the value of the cosmological constant.

Summarizing, casting ordinary general relativity as a perturbative theory in $\Lambda^{-1}$ and starting with background theory in which $\Lambda \rightarrow \infty$ allows to explicitly compute non-degenerate physical quantum states and observables. The technique is applicable in the theory in general and also in minisuperspace models. In the general theory, the calculations are well defined and tractable, but quite involved. This opens up a program for the quantization of the gravitational field that is well defined computationally and allows contact with the physical observables of the theory, a key ingredient in the road to finding a theory with a semiclassical regime that makes contact with ordinary low energy physics.

We wish to thank Abhay Ashtekar and Karel Kuchař for discussions. This work was supported in part by the National Science Foundation under grants PHY-9423950, INT-9811610, PHY-9407194, research funds of the Pennsylvania State University and the Eberly Family research fund at PSU. We acknowledge support of PEDECIBA.

[1] T. Thiemann, Class. Quan. Grav. 15, 839; 15, 875; 15, 1207; 15, 1249; 15, 1281; 15, 1463 (1998).

[2] C. Di Bartolo, R. Gambini, J. Griego and J. Pullin, Phys. Rev. Lett. 84, 2314 (2000); see also gr-qc/9911009; grqc/9911010

[3] A. Ashtekar and J. Lewandowski, J. Math. Phys. 36, 2170 (1995); "Differential geometry on the space of connections via graphs and projective limits," J. Geom. Phys. 17, 191 (1995).

[4] C. Rovelli and L. Smolin, Nucl. Phys. B442, 593 (1995).

[5] C. Rovelli, in http://www.livingreviews.org

[6] B. S. Dewitt, Phys. Rev. 160, 1113 (1967).

[7] C. G. Torre and I. M. Anderson, Phys. Rev. Lett. 70, 3525 (1993).

[8] L. Smolin, gr-qc/9609034

[9] L. Smolin, gr-qc/9301016.

[10] C. Rovelli, J. Math. Phys. 36, 6529 (1995).

[11] Rescaling the constraint implies rescaling the lapse. In the $\Lambda \rightarrow \infty$ limit this means a vanishing lapse. Our theory is therefore similar to the Husain-Kuchař theory, but with a cosmological constant. V. Husain, K. Kuchaŕ, Phys. Rev. Phys. Rev. D42, 4070 (1990).

[12] C. Rovelli, Nucl. Phys. B405, 797 (1993); V. Husain, Phys. Rev. D47, 5394 (1993);

[13] L. Smolin, Phys. Rev. D49, 4028 (1994).

[14] P. J. Arias, C. Di Bartolo, X. Fustero, R. Gambini and A. Trias, Int. J. Mod. Phys. A7, 737 (1992).

[15] A. Ashtekar and J. Lewandowski, Adv. Theor. Math. Phys. 1, 388 (1998); T. Thiemann, J. Math. Phys. 39, 3347 (1998).

[16] R. Gambini, J. Pullin, "The large cosmological constant approximation to classical and quantum gravity: model examples, gr-qc/0008032 (2000).

[17] A. Ashtekar and J. Pullin, Ann. Isr. Phys. Soc. 9, 65-77 (1990).

[18] S. Hervik, gr-qc/0003084. 


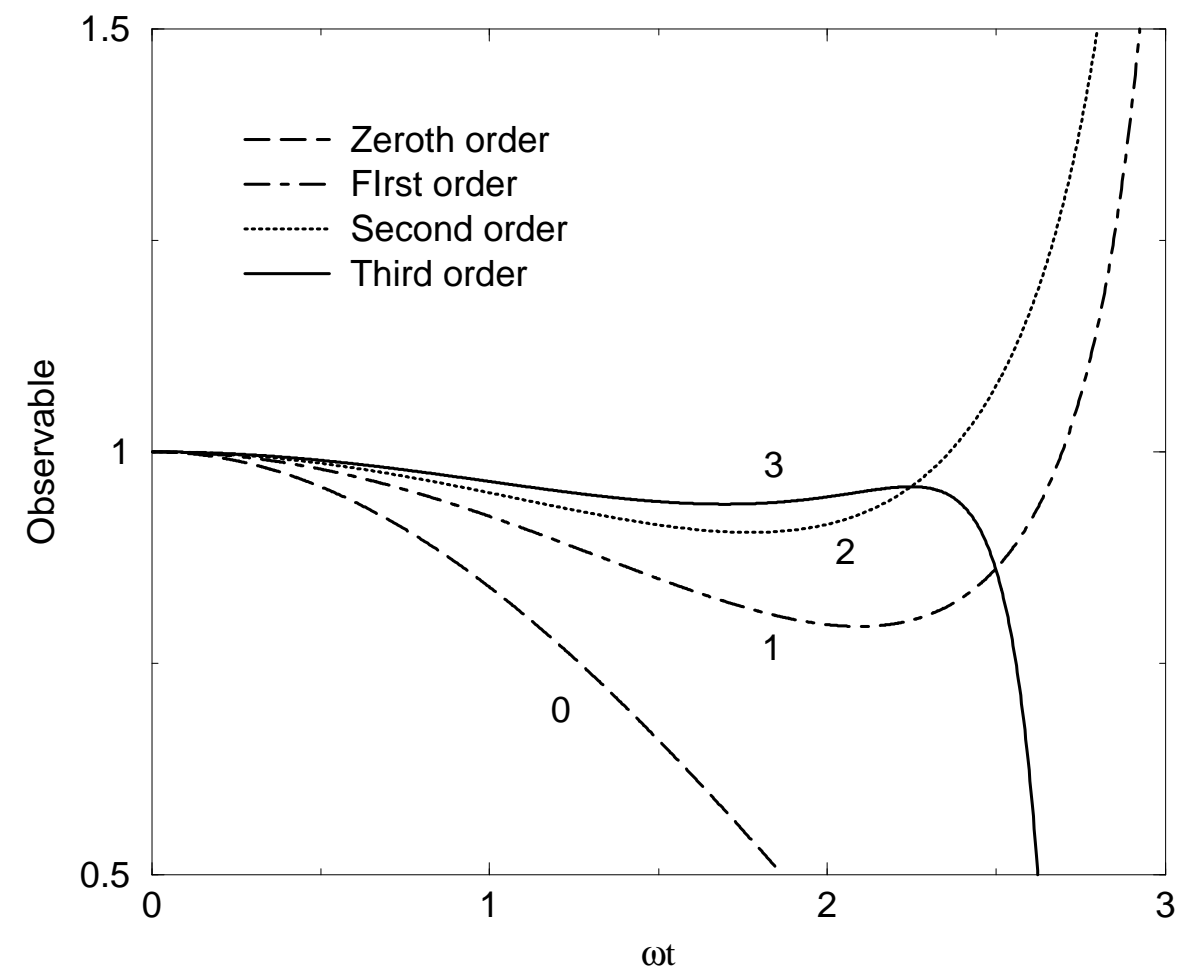

FIG. 1. The perturbative observable evaluated for a Bianchi I cosmology with negative cosmological constant. The time runs from zero, the Big Bang, to $\pi$, the Big Crunch. The four curves correspond to better and better approximations. 